\title{
NOTE ON EXTREME FORMS
}

\author{
E. S. BARNES
}

Let $f\left(x_{1}, \ldots, x_{n}\right)=\sum a_{i j} x_{i} x_{j}$ be a positive definite quadratic form of determinant $D=\left|a_{i j}\right|$, and let $M$ be the minimum of $f$ for integral $x_{1}, \ldots, x_{n}$ not all zero. The form $f$ is said to be extreme if the ratio $M^{n} / D$ does not increase when the coefficients $a_{i j}$ of $f$ suffer any sufficiently small variation.

All extreme forms in $n$ variables are known for $n \leqslant 5$. Hofreiter (2) investigated the problem of finding all extreme forms in 6 variables and listed four forms; but, as is pointed out by Coxeter (1), one of these $\left(F_{4}\right)$ is certainly not extreme. Coxeter (1) actually finds independently four extreme forms (including three of the four listed by Hofreiter) and makes the reasonable suggestion that the list is now complete.

The main purpose of this note is to show that there is an extreme form in 6 variables not given by these authors, namely

$$
f\left(x_{1}, \ldots, x_{6}\right)=\left(\sum_{i=1}^{6} x_{i}\right)^{2}+\sum_{j=1}^{3} \phi\left(x_{j}, x_{3+j}\right),
$$

where generally

for which

$$
\phi(x, y)=x^{2}-x y+y^{2}
$$

$$
M=2, D=\frac{13 \cdot 3^{3}}{2^{6}}
$$

The form (1) is the particular case $n=2 r=6$ of the form

$$
f\left(x_{1}, \ldots, x_{n}\right)=\left(\sum_{i=1}^{n} x_{i}\right)^{2}+\sum_{j=1}^{r} \phi\left(x_{j}, x_{r+j}\right)+\sum_{k=2 r+1}^{n} x_{k}{ }^{2},
$$

where $n \geqslant 2 r \geqslant 2$ (and the last sum is empty if $n=2 r$ ). I show here that the form (2) is extreme if and only if

$$
4 r-2 \geqslant n \geqslant 2 r \geqslant 6 .
$$

We first examine all integral sets $x_{1}, \ldots, x_{n}$, not all zero, for which

$$
f \leqslant 2 \text {. }
$$

Noting that

$$
\phi(x, y)\left\{\begin{array}{l}
=0 \text { if } \quad(x, y)=(0,0), \\
=1 \text { if } \pm(x, y)=(1,0),(0,1) \text { or }(1,1), \\
\geqslant 3 \text { otherwise, }
\end{array}\right.
$$

Received October 1, 1954. The author would like to thank Professor Coxeter and Professor Mordell for their comments on an earlier draft of this note. 
we see that (4) implies that

$$
\phi\left(x_{j}, x_{r+j}\right) \leqslant 1 \quad(j=1, \ldots, r),
$$

and that equality can hold in (6) for at most two values of $j$.

If, say, $\phi\left(x_{1}, x_{r+1}\right)=\phi\left(x_{2}, x_{r+2}\right)=1, \phi\left(x_{j}, x_{r+j}\right)=0(j \geqslant 3)$, then

$$
x_{j}=x_{r+j}=0(3 \leqslant j \leqslant r),
$$

and (4) requires $2 f=2, x_{k}=0(k \geqslant 2 r+1), x_{1}+x_{2}+x_{r+1}+x_{r+2}=0$.

Using (5), we see that all possible sets $\left(x_{1}, x_{2}, x_{r+1}, x_{r+2}\right)$ are $\pm(1,-1,0,0)$, $\pm(1,0,0,-1), \pm(0,1,-1,0), \pm(0,0,1,-1), \pm(1,-1,1,-1)$.

If only one $\phi\left(x_{j}, x_{r+j}\right)$ is non-zero, say $\phi\left(x_{1}, x_{r+1}\right)$, then $x_{j}=x_{r+j}=0$ $(2 \leqslant j \leqslant r)$ and $x_{2 r+1}{ }^{2}+\ldots+x_{n}{ }^{2} \leqslant 1$. There are thus two possibilities:

(i) $x_{2 r+1}=\ldots=x_{n}=0, \quad f=2 x_{1}{ }^{2}+2 x_{r+1}{ }^{2}+x_{1} x_{r+1}$,

and trivially $f \geqslant 2$, with equality only if

$$
\left(x_{1}, x_{r+1}\right)= \pm(1,0), \pm(0,1) \text {; }
$$

(ii) $x_{k}= \pm 1$ for some $k \geqslant 2 r+1$,

$$
f=\left(x_{1}+x_{r+1}+x_{k}\right)^{2}+2,
$$

and so $f \geqslant 2$, with equality only if

$$
\left(x_{1}, x_{r+1}, x_{k}\right)= \pm(1,0,-1), \pm(0,1,-1) .
$$

If finally all $\phi\left(x_{j}, x_{r+j}\right)$ are zero, then

$$
f=\left(\sum_{k=2 r+1}^{n} x_{k}\right)^{2}+\sum_{k=2 r+1}^{n} x_{k}^{2},
$$

and it is easily seen that $f \geqslant 2$, with equality only if some $x_{k}$ is \pm 1 and the rest zero, or if some pair $\left(x_{k}, x_{l}\right)= \pm(1,-1)$ and the remaining $x_{k}$ are zero.

We have thus shown that $M=2$. Also, if with each pair $\pm\left(x_{1}{ }^{(t)}, \ldots x_{n}{ }^{(t)}\right)$ of minimal vectors (i.e., sets with $f=2$ ), we associate the linear form

$$
\lambda_{t}=\lambda_{t}\left(y_{1}, \ldots, y_{n}\right)=\sum_{i=1}^{n} x_{i}{ }^{(t)} y_{i}
$$

we see (using the obvious symmetries of $f$ ) that there are precisely

$$
s=\frac{1}{2} n(n+1)+\frac{1}{2} r(r-3)
$$

such linear forms, given by

$$
\begin{aligned}
& y_{i} \\
& y_{j}-y_{k} \\
& y_{l}-y_{m}+y_{r+l}-y_{r+m}
\end{aligned}
$$

If $r \geqslant 3$, it is easily verified that $f$ is uniquely determined by the fact that it has minimum 2 and associated linear forms (8); thus $f$ is perfect (in the sense of Voronoï (3)). If however $r<3$, (7) gives $s<\frac{1}{2} n(n+1)$ and so $f$ 
cannot be perfect. We have therefore shown that $f$ is perfect if and only if $r \geqslant 3$.

Now Voronoï (3) has shown that a form is extreme if and only if it is perfect and eutactic. We therefore consider next the problem of deciding when $f$ is eutactic, that is to say, when its adjoint $F\left(y_{1}, \ldots, y_{n}\right)$ is expressible as

$$
F\left(y_{1}, \ldots, y_{n}\right)=\sum_{t=1}^{s} \rho_{t} \lambda_{t}{ }^{2}, \rho_{t}>0 \quad(t=1, \ldots, s) .
$$

The labour of calculating $F$ (and the determinant $D$ of $f$ ) may be lightened by using the following method:

The form

$$
g\left(z_{1}, \ldots, z_{n}\right)=\left(\alpha_{1} z_{1}+\ldots+\alpha_{n} z_{n}\right)^{2}+\sum_{i=1}^{n} z_{i}{ }^{2}
$$

is easily found to have determinant

$$
D(g)=1+\sum_{i=1}^{n} \alpha_{i}^{2}
$$

and adjoint a multiple of

$$
G\left(z_{1}, \ldots, z_{n}\right)=\sum c_{i j} z_{i} z_{j}
$$

with

$$
c_{i i}=1+\sum_{\substack{k=1 \\ k \neq i}}^{n} \alpha_{k}^{2}, c_{i j}=-\alpha_{i} \alpha_{j} \quad(i \neq j)
$$

Under the linear transformation $T$ defined by

$$
\left.\begin{array}{rl}
x_{j} & =z_{j}+(1 / \sqrt{ } 3) z_{r+\jmath} \\
x_{r+\jmath} & =(2 / \sqrt{ } 3) z_{r+\jmath} \\
x_{k} & =z_{k}
\end{array}\right\} \quad \begin{array}{r}
(j=1, \ldots, r), \\
(k=2 r+1, \ldots, n),
\end{array}
$$

$f$ in (2) is reduced to the form (10) with

(13) $\alpha_{i}=1 \quad(1 \leqslant i \leqslant r, 2 r+1 \leqslant i \leqslant n), \quad \alpha_{i}=\sqrt{ } 3 \quad(r+1 \leqslant i \leqslant 2 r)$, so that

$$
1+\sum_{i=1}^{n} \alpha_{i}^{2}=n+2 r+1
$$

Since $T$ has determinant $(2 / \sqrt{ } 3)^{r}$, it follows from (11) and (14) that $f$ has determinant

$$
D=\left(\frac{3}{4}\right)^{r}(n+2 r+1) .
$$

Finally, a straightforward multiplication of matrices now shows that $F\left(y_{1}, \ldots, y_{n}\right)$ is a multiple of $\sum b_{i j} y_{i} y_{j}$ with 


$$
\begin{aligned}
b_{i i} & = \begin{cases}4(n+2 r-2), & 1 \leqslant i \leqslant 2 r, \\
3(n+2 r), & i>2 r,\end{cases} \\
b_{i j} & = \begin{cases}-12, & 1 \leqslant i<j \leqslant 2 r, j-i \neq r, \\
2(n+2 r-5), & 1 \leqslant i<j \leqslant 2 r, j-i=r, \\
-6, & i \leqslant 2 r, j>2 r, \\
-3, & j>i>2 r .\end{cases}
\end{aligned}
$$

Corresponding to the enumeration (8) of the associated linear forms, we write (9) as

$$
\begin{aligned}
F\left(y_{1}, \ldots, y_{n}\right) & =\sum \rho_{i} y_{i}{ }^{2}+\sum \sigma_{j k}\left(y_{j}-y_{k}\right)^{2} \\
& +\sum \tau_{l m}\left(y_{l}-y_{m}+y_{r+l}-y_{r+m}\right)^{2},
\end{aligned}
$$

where the suffixes have the ranges given in (8), and solve (17) for the $s=\frac{1}{2} n(n+1)+\frac{1}{2} r(r-3)$ coefficients $\rho_{i}, \sigma_{j k}, \tau_{l m}$.

First, we have immediately

$$
\begin{array}{rlrl}
\sigma_{j k} & =-b_{j k}=3, & & k>j>2 r, \\
\sigma_{j k} & =-b_{j k}=6, & k>2 r \geqslant j .
\end{array}
$$

The coefficient of $2 y_{j} y_{k}$ for $1 \leqslant j<k \leqslant 2 r, k-j \neq r$ is $-\sigma_{j k}-\tau_{l m}$, where $l=j$ or $j-r, m=k$ or $k-r$; hence we have

$$
\sigma_{j k}=\sigma_{j, r+k}=\sigma_{r+\jmath, r+k}=12-\tau_{j k}, \quad 1 \leqslant j<k \leqslant r .
$$

The coefficient of $2 y_{j} y_{r+j}$, for $1 \leqslant j \leqslant r$, is

$$
\tau_{i j}+\ldots+\tau_{j-1, j}+\tau_{j, j+1}+\ldots+\tau_{j r}=2(n+2 r-5)
$$

The coefficient of $y_{1}^{2}$ is

$$
(j=1, \ldots, r) \text {. }
$$

$$
\begin{aligned}
\rho_{1}+ & \sigma_{12}+\ldots+\sigma_{1 n}+\tau_{12}+\ldots+\tau_{1 r} \\
= & \rho_{1}+\left(12-\tau_{12}\right)+\ldots+\left(12-\tau_{1 r}\right)+\left(12-\tau_{12}\right)+\ldots+\left(12-\tau_{1 r}\right) \\
& \quad+6(n-2 r)+\tau_{12}+\ldots+\tau_{1 \tau} \\
= & \rho_{1}+12(2 r-2)+6(n-2 r)-\left(\tau_{12}+\ldots+\tau_{1 r}\right) \\
= & \rho_{1}+4 n+8 r-14,
\end{aligned}
$$

using (19), (20) and (21); since $b_{11}=4(n+2 r-2)$, it follows that $\rho_{1}=6$. The same argument gives

$$
\rho_{i}=6 \quad 1 \leqslant i \leqslant 2 r .
$$

The coefficient of $y_{2 r+1}{ }^{2}$ is

$$
\begin{aligned}
\rho_{2 r+1}+\sigma_{1,2 r+1}+\ldots+\sigma_{2 r, 2 r+1}+\sigma_{2 r+1,2 r+2}+\ldots+\sigma_{2 r+1, n} \\
\\
=\rho_{2 r+1}+6(2 r)+3(n-2 r-1),
\end{aligned}
$$

using (18) and (19); since $b_{2 r+1,2 \tau+1}=3(n+2 r)$, it follows that $\rho_{2 \tau+1}=3$. The same argument gives

$$
\rho_{i}=3, \quad 2 r+1 \leqslant i \leqslant n .
$$


Now (20) and (21) give

$$
\begin{aligned}
\sigma_{12}+\ldots+\sigma_{1 r} & =\left(12-\tau_{12}\right)+\ldots+\left(12-\tau_{1 r}\right) \\
& =12(r-1)-2(n+2 r-5) \\
& =2(4 r-1-n) ;
\end{aligned}
$$

if the $\sigma_{i j}$ are all strictly positive, this shows that $n<4 r-1$. Thus $f$ is not eutactic if $n \geqslant 4 r-1$.

If, however, $n \leqslant 4 r-2$ and $r \geqslant 3$, we can show that $f$ is eutactic by taking the particular solution

$$
\tau_{l m}=\frac{2(n+2 r-5)}{r-1} \quad(1 \leqslant l<m \leqslant r)
$$

of the $r$ equations (21). Then (20) gives

$$
\sigma_{j k}=12-\frac{2(n+2 r-5)}{r-1}=\frac{2(4 r-1-n)}{r-1}>0
$$

for all relevant $j, k$, and we have exhibited a solution of (17) in which all the coefficients $\rho_{i}, \sigma_{j k}, \tau_{l m}$ are positive.

We have now established our assertion that $f$ is extreme if and only if (3) holds. In particular, we have shown that the senary form (1), for which $n=2 r=6$, is extreme.

The form (2) gives some information on the possible structure of perfect, eutactic and extreme forms, as well as extending Coxeter's table (1, p. 439) of extreme forms for each $n \geqslant 6$.

Thus Coxeter remarks (1, p. 396): "For every known perfect form in less than nine variables there is a solution [of (9)] with the $\rho$ 's all equal." However, for the form (2), there is no such solution for any $n \geqslant 2 r \geqslant 6$. This assertion is clear if $n>2 r$, from (22) and (23); if $n=2 r$, it follows from (24) and (25), since equality of the $\tau$ 's and $\sigma$ 's would require

$$
4 r-5=2 r-1, r=2 .
$$

Coxeter also remarks (1, p. 392): "We do not know whether every perfect form is extreme." The form (2), however, is perfect and non-eutactic (and so not extreme) for any $r \geqslant 3, n \geqslant 4 r-1$.

\section{REFERENCES}

1. H. S. M. Coxeter, Extreme forms, Can. J. Math. 3 (1951), 391-441.

2. N. Hofreiter, Ueber Extremformen, Monatsh. Math. Phys. 40 (1933), 129-152.

3. G. Voronoï, Sur quelques propriétés des formes quadratiques positives parfaites, J. reine angew. Math. 193 (1908), 97-178.

\section{University of Sydney, Australia}

